\begin{tabular}{|c|c|c|c|c|}
\hline Variable & Patient 1 & Patient 2 & Patient 3 & Patient 4 \\
\hline \multicolumn{5}{|l|}{ Clinical features } \\
\hline Age (year) & 17.1 & 15.4 & 8.5 & 14.4 \\
\hline Sex & Female & Male & Male & Female \\
\hline Indication & Lactic acidosis & $\begin{array}{l}\text { Sepsis/Cytokines } \\
\text { storm }\end{array}$ & Hyperbilirubinaemia & Rhabdomyolysis \\
\hline PIM3 predicted mortality (\%) & 6.2 & 31.4 & 5.9 & 17.1 \\
\hline Pre-therapy eGFR $\left(\mathrm{ml} / \mathrm{min} / 1.73 \mathrm{~m}^{2}\right)$ & 210.2 & 133.8 & 45.6 & \\
\hline Need for mechanical ventilation & No & Yes & No & Yes \\
\hline Need for inotropes & No & Yes & No & Yes \\
\hline \multicolumn{5}{|l|}{ EBP/CRRT parameters } \\
\hline Technique & $\begin{array}{l}\text { High volume } \\
\text { haemodiafiltration }\end{array}$ & Haemoperfusion & $\begin{array}{l}\text { Single-pass albumin } \\
\text { dialysis }\end{array}$ & Haemoperfusion \\
\hline Prior conventional CRRT & No & No & Yes & Yes \\
\hline Filter & ST100 & Oxiris & ST100 & Cytosorb \\
\hline Mean blood flow rate $(\mathrm{ml} / \mathrm{kg} / \mathrm{min})$ & 4.7 & 4.1 & 3.7 & \\
\hline $\begin{array}{l}\text { Mean replacement rate }(\mathrm{ml} / \mathrm{kg} / \\
\text { hour) }\end{array}$ & 21.4 & 32.5 & 23.0 & \\
\hline Mean dialysate rate $(\mathrm{ml} / \mathrm{kg} / \mathrm{hour})$ & 72.7 & 32.2 & 30.3 & \\
\hline Anti-coagulation & Heparin & Nil & Nil & Heparin \\
\hline Treatment duration (hour) & 160.9 & 37.0 & 42.1 & 8.0 \\
\hline
\end{tabular}

Results Altogether four patients were identified during the period (table 1). No major CRRT-specific complication was encountered except mild electrolytes disturbances.

Patient 1: A 17-year-old girl received high-volume haemodiafiltration as a bridging therapy for her refractory type B lactic acidosis related to the relapse of B-cell acute lymphoblastic leukaemia (ALL). She had a peak lactate level of 18 $\mathrm{mmol} / \mathrm{L}$ with lowest $\mathrm{pH} 7.13$ and bicarbonate level $6.0 \mathrm{mmol} /$ L. The measured mean lactate clearance was $65 \mathrm{ml} / \mathrm{kg} /$ hour.

Patient 2: A 15-year-old male with T-cell ALL had peritonitis and pneumoperitoneum complicated with Klebsiella pneumoniae septicaemia. He required high dose inotropes despite anti-microbials and intravenous immunoglobulin. Two sessions of haemoperfusion using the Oxiris ${ }^{\circledR}$ filter were then performed for endotoxin removal. The inotropes were successfully weaned off afterwards. Resection of the remaining necrotic small bowel was performed 3 days later.

Patient 3: An 8-year-old boy with bone marrow transplantation gradually developed conjugated hyperbilirubinaemia due to acute veno-occlusive disease and graft-versus-host disease. He received a session of single-pass albumin dialysis using 4\% albumin as dialysate to reduce his bilirubin level, bridging him to receive a liver biopsy that confirmed his diagnosis. The total bilirubin level decreased from 305 to $222 \mu \mathrm{mol} / \mathrm{L}$ after the therapy.

Patient 4: A 14-year-old girl with undiagnosed anaplastic large cell lymphoma was admitted for refractory shock that deteriorated rapidly causing multi-organ failure. She was then started on veno-arterial extracorporeal membrane oxygenation but was complicated with reperfusion injury causing rhabdomyolysis with a peak creatine kinase (CK) level up to 264500IU/L. Myoglobulin clearance using the absorbent Cytosorb ${ }^{\boxplus}$ was then added to the CRRT. Her CK level was successfully brought down to $97436 \mathrm{IU} / \mathrm{ml}$ after 8 hours of therapy. However, she did not respond to the chemotherapy and finally succumbed 5 days after admission.

Conclusions EBP using different techniques and filters can be safely applied in selected children as an adjunctive therapy for various conditions. However, the optimal dose, timing of initiation and monitoring target remain largely unanswered.

\section{A REVIEW ON PAEDIATRIC PALLIATIVE CARE PROGRAM IN A REGIONAL HOSPITAL IN HONG KONG}

Tracy Yuen Han Lee, Sze Sun Man, Choi Ha Kwong, Chak Ho Li. Hong Kong

\subsection{6/bmjpo-2021-RCPCH.235}

Background Paediatric palliative care (PPC) focuses on the enhancement of quality of life for children with life-limiting diseases and support to their families. This study reviewed the paediatric palliative care program in Hong Kong (HK).

Objectives Reviewed the characteristics and service need of children receiving PPC in a regional hospital in HK.

Methods This is a retrospective chart review. All children aged less than 18 years old receiving PPC at a regional hospital in HK from 2015 to 2020 were included.

Results Fifty-eight children received PPC service in the study period. Eight cases were referred for bereavement support after death and were excluded. A total of 50 children (M : F $=24: 26$ ) were enrolled. Median age of referral to PPC service was 8 years 9 months. Average duration of follow up was 1 year 4 months (median 9 months). Underlying diseases included neurological diseases $n=20(40 \%)$, congenital malformations and chromosomal anomalies $n=10$ (20\%), neoplasms $\mathrm{n}=10$ (20\%), endocrine and metabolic conditions $\mathrm{n}=7(14 \%)$, perinatal conditions $\mathrm{n}=2(4 \%)$ and external causes of morbidity $\mathrm{n}=1(2 \%)$. Sources of referral included intensivists $(32 \%)$, general paediatricians $(30 \%)$, oncologists $(14 \%)$, neonatologists (12\%), neurologists (8\%), endocrinologists (2\%) and respirologists $(2 \%)$.

Thirty-nine patients $(78 \%)$ resided in the community (home $48 \%$, residential schools $30 \%)$. Majority of children $(60.8 \%)$ had high medical needs during their daily care, including wheelchair-bound $n=34(66.7 \%)$, gastrostomy feeding $n=17$ 
(33.3\%), nasogastric tube-feeding $\mathrm{n}=8$ (15.7\%), non-invasive ventilatory support $n=13(25.5 \%)$ and tracheostomy with ventilatory support $\mathrm{n}=5(9.8 \%)$.

Advanced care plan (ACP) was discussed with 25 (50\%) families. Among the 18 cases who died while receiving PPC, Do-Not Attempt Cardiopulmonary Resuscitation (DNACPR) was discussed with 15 (83.3\%) of them. DNACPR had been discussed with $37.5 \%$ of the surviving cases. Median time from referral to death varied with the diseases: neoplasms (19 days), neurological conditions (54 days), congenital malformations and chromosomal anomalies (65 days), endocrine and metabolic conditions (174 days), external causes of morbidity (424 days). Forty-five percent of children passed away in the general paediatric wards (38.9\%), Accident and Emergency Department $(5.6 \%)$ while the remaining children passed away in the Intensive Care Units (44.4\% in Paediatric ICU, $11.1 \%$ in Neonatal ICU). No patient died at home. Pain control medications were given during end-of-life period in $30.8 \%$ of cases.

Conclusions This review showed majority of children on palliative care stayed at home or residential schools and had high medical needs. Community support to these families was important. The time from referral to death was short. Advanced Care Plan was discussed with only half of the families. Less than one-third of patients received pain control at end-of-life. Paediatric palliative care service is under-developed and has to be largely promoted in Hong Kong.

\section{MOM, I CAN'T BREATHE!}

Khoironi Omar, Siew Sing Chua, Amelia Alias, Noraini Ab Rahman, Fong Chiao Lai. Malaysia

\subsection{6/bmjpo-2021-RCPCH.236}

Background Sinus of Valsalva aneurysm (SVA) is a rare cardiac anomaly, either acquired or congenital. This anomaly most often arises from the right coronary sinus, while less frequently arises from the non-coronary sinus or left sinuses. Ruptured sinus of Valsalva (RSOV), especially if it arises from the right coronary sinus, can cause acute symptoms of right sided heart failure. This is mainly because RSOV from right coronary sinus will lead to communication between aorta and right atrium or right ventricle, leading to a left to right shunt, hence causing right ventricle overload. RSOV can be an isolated lesion or associated with other cardiac lesions e.g. ventricular septal defect (VSD), aortic regurgitation, bicuspid aortic valve, and coarctation of aorta. RSOV rarely occurs in infancy and childhood; it mostly occurs after puberty or between 30's to 40's. Early and accurate diagnosis with early surgical intervention is the only way to improve survival in patient with RSOV.

Objectives To provide a review on our detection and management on RSOV.

Methods This is a case report of a teenager who was premorbid well, presented to a district hospital with acute heart failure symptoms.

Results A 14-year-old girl presented with chest pain, orthopnoea and reduced effort tolerance for 4 days. Physical examination revealed pansystolic murmur grade 4 with no hyperactive precordium. The electrocardiogram showed a normal rhythm with sinus tachycardia. Chest imaging showed neither any lung infection nor cardiomegaly. From the preliminary echocardiography, there was presence of turbulence flow in the aortic root with small ventricular septal defect. Ruptured SVA was highly being suspected in this patient. She was started on anti-failure medications. Further assessment was done at tertiary centre by Paediatric Cardiologist, confirming the diagnosis of RSOV. Patient was referred to cardiothoracic team for repair and was discharged well without any post-operative complications.

Conclusions RSOV is a very rare cause of acute heart failure in paediatrics population. RSOV should be suspected in patient with small VSD on follow up, especially if patient present with acute heart failure. The diagnosis requires a high index of suspicion from history, to presentation, to investigation, such as echocardiography. It is a surgical emergency and early surgery is indicated.

\section{ENSURING SAFE USE OF PRESSURISED METERED DOSE INHALERS WITHOUT A BUILT-IN DOSE COUNTER}

Soh Yan Tan, Mary Hoon, Yi Hua Tan, Ai Huay Teo, Zai Ru Cheng, Oon Hoe Teoh. Singapore

\subsection{6/bmjpo-2021-RCPCH.237}

Background Most patients using pressurised metered dose inhalers (pMDIs) without a built-in dose counter often arbitrarily gauge the doses left in the canister using imprecise methods. As the delivered dose becomes variable beyond the labelled maximum dose for pMDIs, this imprecise practice may lead to patients receiving sub-therapeutic doses and putting their lives at risk, especially for reliever medication. Subtherapeutic doses of controller medication may adversely affect asthma control. In some situations, patients may be discarding their pMDIs too early.

Objectives We propose a simple and cost-effective method to ensure safe use of pMDIs without a built-in dose counter.

Methods We created customised adhesive count stickers with running numbers up to the maximum dose for various pMDIs that can be pasted on the inhalers. Patients would struck off a number after the administration of a dose on the sticker, and were therefore able to keep track of the doses left on the pMDI. They were instructed to change to a new pMDI once the numbers on the count stickers were fully struck off. We compared the weight of the empty/used pMDIs collected from patients before and after the introduction of adhesive count stickers, to objectively assess if patients were changing to new inhalers too early or too late, and determine the effectiveness of the adhesive count stickers.

Results A total of 200 empty/used pMDIs were collected from patients both pre-intervention and post-intervention. Our results showed statistical significant differences in the weight of the canisters collected prior to and after the intervention. The median weight of the salbutamol pMDIs collected from patients pre-intervention was $9.495 \mathrm{~g}$ and postintervention was $11.075 \mathrm{~g}$. The median weight of the fluticasone diproprionate pMDIs collected from patients pre-intervention was $9.745 \mathrm{~g}$ and post-intervention was $12.145 \mathrm{~g}$. There is statistically significant safety margin of $1.58 \mathrm{~g}$ and $2.40 \mathrm{~g}$ for each category of pMDI respectively. The adhesive count stickers were well received by the patients with good feedback.

Conclusions Our results suggest that without objective methods of dose counting, patients are using their pMDIs beyond the 